\title{
PAREAMENTO AO MODELO COM AJUSTE DO ATRASO: UMA REVISÃO DA LITERATURA
}

\author{
TITRATING DELAYED MATCHING-TO-SAMPLE: A LITERATURE REVIEW
}

\author{
CHARLISE ALBRECHT - ORCID 0000-0001-9465-8185 \\ UNIVERSIDADE DE BRASÍLIA, BRASIL \\ ELENICE S. HANNA - ORCID 0000-0002-5222-1847 \\ UniversidAde de Brasília E INSTITUTO NACIONAL DE CIÊNCIA E TECNOLOGIA SOBRE COMPORTAMENTO COGNIÇÃo E ENSINO, BRASIL
}

\section{RESUMO}

O Pareamento-ao-Modelo com Ajuste do Atraso (TDMTS) é utilizado para estudar memória de trabalho e fenômenos correlatos. Neste procedimento, a escolha do estímulo correspondente ao modelo deve ocorrer após um intervalo de tempo desde a retirada do modelo (atraso). $\mathrm{O}$ atraso é ajustado a partir dos acertos e erros do participante. $\mathrm{O}$ objetivo deste artigo foi fazer uma revisão de literatura de estudos que manipularam variáveis metodológicas do TDMTS. A partir de buscas em bancos de dados, na internet e nas referências dos artigos encontrados, foram analisados 16 artigos, que descrevem 21 experimentos, publicados de 1965 a 2019. A revisão mostrou os critérios e valores de parâmetros mais utilizados na literatura, e resumiu os efeitos da manipulação de aspectos metodológicos. Atrasos ajustados mais longos estão relacionados ao: aumento da observação ao modelo, programação do atraso inicial de cada sessão baseada nos atrasos finais da sessão anterior; critério de ajuste mais tolerante; maiores valores do incremento; e programação de reforços diferenciais para cada discriminação. Estes resultados carecem de replicações controladas para avaliar a generalidade, considerando a variedade de parâmetros, critérios e sujeitos experimentais.

Palavras-chave: discriminação condicional, memória de trabalho, pareamento-ao-modelo com atraso, ajuste do atraso, revisão.

\begin{abstract}
Titrating Delayed Matching-to-Sample (TDMTS) is used to investigate working memory and correlates. In this procedure, response that matches the sample is emitted after a time interval since the sample withdrawal (delay). Delay is adjusted based on correct and incorrect responses. The present paper presents a literature review of studies that manipulated methodological variables of TDMTS. From search in data bases, internet and references of the papers, 16 articles published from 1965 to 2019, that describe 21 experiments, were analyzed. This review showed the most used criteria and parameters values in the literature and summarized the effects of methodological aspects manipulated in studies. Longer adjusted delays were found to be related to: larger response ratios on sample; initial session delay setting based on previous session last values; more tolerant adjusting criteria; larger step sizes; and programming differential reinforcement for each discrimination. Those results call for controlled replications to assess generality, considering the variety of parameters, criteria and experimental subjects.
\end{abstract}

Keywords: conditional discrimination, working memory, titrating delayed matching-to-sample, review

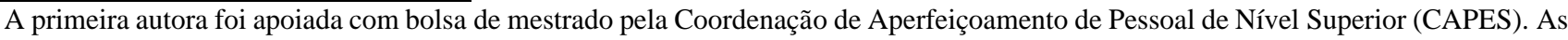
autoras são filiadas ao Instituto Nacional de Ciência e Tecnologia sobre Comportamento, Cognição e Ensino, INCT-ECCE (Deisy G. de Souza, presidente) financiado pelo CNPq (\#573972/2008-7) e FAPESP (2008/57705-8). Correspondência referente a este artigo deverá ser endereçada para Elenice S. Hanna, Departamento de Processos Psicológicos Básicos, Instituto de Psicologia, Universidade de Brasília, Campus Darcy Ribeiro, ICC Sul, Sala ASS-015, Brasília, DF, Brasil. E-mail: elenicehanna@gmail.com
}

DOI: http://dx.doi.org/10.18542/rebac.v15i1.8327 
Os processos de atentar, perceber e lembrar podem ser analisados como relações entre aspectos do ambiente e do comportamento, ou seja, relações de controle de estímulos (Matos, 1981). Um comportamento está sob controle de estímulos quando é provável que respostas ou classes de respostas ocorram na presença de determinados estímulos e não de outros (Catania, 1999). Quando o controle de estímulos é estabelecido pode-se afirmar que o organismo está discriminando ou diferenciando duas ou mais situações. A relação entre o responder discriminado e tais estímulos pode ser controlada por outros estímulos, gerando um processo comportamental chamado de discriminação condicional (Sidman, 1986).

As discriminações condicionais - tipo de discriminação na qual as respostas são reforçadas na presença de um determinado estímulo se, e somente se, outra condição estiver presente - podem ser ensinadas por meio de um procedimento experimental denominado matching-to-sample (MTS) (Cumming \& Berryman, 1965), expressão traduzida como pareamento ao modelo, emparelhamento de acordo com o modelo ou emparelhamento com o modelo.

O MTS é um procedimento de tentativas sucessivas discretas. Em uma tentativa, um estímulo modelo (condicional) é apresentado ao participante, que pode ou não ter que emitir respostas de observação (e.g., apontar). Em seguida são apresentados dois ou mais estímulos de comparação (discriminativos), cujas funções são definidas a depender do estímulo condicional apresentado (Sidman, 1986). O participante deve escolher o estímulo de comparação correspondente ao estímulo modelo, que funciona como um seletor de discriminações (Cumming \& Berryman, 1965). A escolha deve ser controlada pelas propriedades dos dois estímulos, uma vez que a consequência é contingente à relação entre os estímulos modelo e discriminativo. A tentativa é finalizada com a apresentação de consequências que indicam se a escolha foi correta ou incorreta e alteram a probabilidade de novas escolhas, conforme as relações entre os estímulos definidas pelo pesquisador.

Podem-se programar versões diferentes do MTS variando-se as relações entre os estímulos que se deseja ensinar e a relação temporal entre os estímulos modelo e comparações. As variações do MTS mais comumente utilizadas para estabelecer relações diferentes entre estímulos modelo e de comparação são: MTS de identidade (escolha reforçada é do estímulo igual ao modelo), MTS do diferente (escolha reforçada é do estímulo diferente do modelo), e MTS arbitrário (escolha reforçada é de um estímulo arbitrariamente definido como correspondente ao modelo) (Mackay, 1991).

Quanto à relação temporal entre a apresentação do estímulo modelo e dos estímulos de comparação, as versões do procedimento são denominadas de MTS simultâneo (Simultaneous Matching-to-Sample - SMTS) e MTS com atraso (Delayed Matching-to-Sample - DMTS) (Mackay, 1991). No SMTS, o estímulo modelo permanece disponível juntamente com os estímulos de comparação. No DMTS, a apresentação dos estímulos de comparação ocorre após a retirada do modelo (e.g., Dube et al., 2006). No DMTS, o atraso pode ser fixo ou variável.

Responder a estímulos de comparação em procedimentos de DMTS pode ser considerado uma forma de lembrar, porque o estímulo modelo, que deveria determinar a escolha do estímulo de comparação, não está mais presente no ambiente (Palmer, 1991). Alguns autores denominam o atraso de intervalo de retenção (e.g., Van Hest \& Steckler, 1996). Manipulações do atraso permitem conclusões sobre o tempo que o modelo ausente ainda exerce controle sobre o comportamento de escolha (Blough, 1959). As versões do MTS que incluem atraso têm sido utilizadas, por isso, para estudar fenômenos relativos ao conceito de memória (Lind, Enquist, \& Ghirlanda, 2015).

O tema principal desta revisão é o TDMTS (Titrating Delayed Matching-to-Sample), que é um tipo especial de MTS com atraso variável, em que o valor do atraso é alterado ao longo das tentativas de acordo com o desempenho do participante. Acertos resultam em aumento no valor do atraso nas tentativas subsequentes, enquanto que erros resultam em diminuição do atraso. Nesse procedimento, o valor do atraso alcançado que mantém desempenho preciso é a principal variável dependente de interesse (Kangas, Vaidya, \& Branch, 2010).

O TDMTS tem se mostrado um procedimento útil para estudar como a duração do atraso influencia o desempenho de discriminações condicionais (o lembrar as relações) em populações clínicas, como indivíduo com demência (e.g., Arntzen \& Steingrimsdottir, 2014) e afásico (e.g., Sidman, Stoddard, Mohr, \& Leicester, 1971), e para acompanhar o progresso de doenças neurodegenerativas (Arntzen \& Steingrimsdottir, 2014). Seu uso tem sido também sugerido em contextos clínicos como ferramenta de diagnóstico de doenças neurodegenerativas e para treinar o comportamento de recordação (Arntzen \& Steingrimsdottir, 2014).

Vantagens do TDMTS em relação ao DMTS com atraso fixo (Fixed Delayed Matching-to-Sample FDMTS) têm sido apontadas. Um procedimento que ajusta o atraso de acordo com o desempenho de cada participante possibilita a criação de sessões personalizadas para diferentes tipos e graus de comprometimentos cognitivos. Além disso, com o TDMTS é possível evitar os efeitos de piso e teto que são comuns no DMTS com atraso fixo (Poling, Temple, \& Foster, 1996). Para Cumming e Berryman (1965), "o TDMTS tem vantagens óbvias para investigações comportamentais de agentes psicofarmacológicos e variáveis motivacionais, por fornecer registro imediato e contínuo da capacidade do sujeito em manter o atraso" (p. 399) ajustado de acordo com o desempenho de cada sujeito.

Apesar das vantagens do procedimento TDMTS citadas, uma recente meta-análise indicou seu uso como menos frequente do que com atraso fixo (Lind et al., 2015). Menos se sabe também sobre os efeitos de variáveis do procedimento e interações de outras variáveis com TDMTS (Kangas et al., 2010).

O TDMTS é um procedimento relativamente complexo. O número de parâmetros e critérios que devem ser definidos é maior do que para o DMTS com atraso 
fixo. Na sua programação, deve-se definir o atraso inicial a ser utilizado, o valor do step size (i.e., tamanho do incremento e decremento de ajuste do atraso), o critério para aumentar ou diminuir o atraso, o restabelecimento (reset) ou não do atraso a cada sessão, como também o critério para calcular o atraso ajustado final. Outros aspectos metodológicos não específicos ao TDMTS se somam à complexidade do procedimento, como o esquema para respostas de observação do modelo, a duração da sessão e o intervalo entre tentativas (IET). Estes parâmetros, atualmente definidos de forma arbitrária pelo experimentador, podem ser relevantes para os resultados dos estudos e, por isso, serão alvo de análise nesse relato.

O objetivo deste artigo foi realizar uma revisão de literatura sobre os estudos que utilizaram o procedimento de TDMTS a partir de 1965 até fevereiro de 2019, e manipularam variáveis metodológicas. A revisão é relevante porque este procedimento permite estudar fenômenos relacionados à memória (controle pelo estímulo que não está mais presente) e quantificar o intervalo de tempo que cada indivíduo é capaz de lembrar do estímulo modelo.

\section{MÉTODO}

As primeiras buscas de registros em bases de dados foram conduzidas em julho de 2018, em três bases de dados: PsycARTICLES, PsycINFO e Web of Science. Foram utilizadas três combinações de termos nas pesquisas: ("matching-to-sample" AND titrating), ("matching-to-sample" AND identity) e "titrating delayed matching-to-sample". No total, 410 artigos foram recuperados (212 foram encontrados na PsycInfo, 184 na Web of Science e 14 na PsycArticles). Depois de excluídos os artigos repetidos (89 artigos), o número de artigos diminuiu para 321. Foram lidos os títulos e os resumos de cada artigo e excluídos 309 artigos que não atenderam três critérios: (1) ser estudo empírico; (2) ter utilizado o procedimento de TDMTS; e (3) não ter manipulado agentes farmacológicos. Depois da exclusão, apenas sete artigos foram mantidos e analisados em seus detalhes. Uma nova pesquisa bibliográfica foi realizada em fevereiro de 2019 a partir das listas de referência destes artigos, do Google Acadêmico (https://scholar.google.com.br) e do Research Gate (https://www.researchgate.net) e resultou na inclusão de mais seis artigos. Desses 13 artigos, um foi eliminado por não incluir no relato as informações metodológicas que buscava-se analisar (Steingrimsdottir, 2015). Por fim, decidiu-se incluir quatro artigos que avaliaram efeito de drogas e manipularam outra variável de interesse para esta análise (IET, step size e idade). O total de artigos analisados foi 16 .

\section{RESULTADOS E DISCUSSÃO}

A Tabela 1 apresenta os 16 artigos, especificando a manipulação realizada, os sujeitos/participantes, o tipo de DMTS utilizado e diversos aspectos do procedimento (estímulos, razão fixa exigida no modelo, valores do atraso inicial e máximo, IET, critério de ajuste do atraso e step size). Três estudos (Friedel, 2011; Kangas et al., 2010; Lian \& Arntzen, 2011) realizaram mais de um experimento com manipulações distintas e, por isso, foram desmembrados na tabela. Para fins da análise apresentada aqui, foram considerados apenas os experimentos de cada artigo que utilizaram TDMTS. No total foram analisados 21 experimentos, apresentados em ordem cronológica.

A análise dos estudos será feita considerando a manipulação realizada. No entanto, foram incluídos estudos de demonstração do controle pelo procedimento de "auto ajuste" que não realizaram manipulações experimentais. Estes estudos foram incluídos por serem pioneiros na utilização do procedimento (Cumming \& Berryman, 1965; Scheckel, 1965) e como instrumento de avaliação (Sidman et al., 1971).

\section{Estudos Pioneiros}

O TDMTS foi descrito primeiramente no estudo de Cumming e Berryman (1965), com pombos, e utilizado no mesmo ano em artigo derivado da tese de Scheckel (1965), com macacos rhesus. Os dois estudos determinaram as curvas de atraso ao longo das sessões de ensino de identidade de cor (escolha do estímulo de comparação igual ao modelo). Duas respostas certas aumentavam o atraso em tentativas subsequentes e um erro reduzia o atraso. Os autores relataram as flutuações que ocorrem nessas curvas de atrasos ao longo da sessão, partindo de atrasos pequenos (mas diferentes entre os estudos) e alcançando atrasos próximos a $12 \mathrm{~s}$ para pombos e $70 \mathrm{~s}$ para macacos rhesus. Os autores tomaram como medida de variabilidade a amplitude dos atrasos, definida como a diferença entre os valores máximos e mínimos de atrasos ajustados. Houve diminuição da amplitude dos valores dos atrasos com o aumento da exposição ao procedimento (ficando em torno de $10 \mathrm{~s}$ para pombos e $45 \mathrm{~s}$ para macacos rhesus). Diversos fatores diferiram entre os procedimentos dos dois estudos. Para aumentar a exposição ao modelo, Cumming e Berryman exigiram cinco respostas no modelo para que os estímulos de comparação fossem apresentados (Razão Fixa 5 - FR 5), enquanto Scheckel exigiu apenas uma resposta no modelo (FR 1) combinado com um Limited Hold 10 s (LH 10; Limite de Espera de $10 \mathrm{~s}$ ), ou seja, a resposta deveria ocorrer em no máximo $10 \mathrm{~s}$ para que a tentativa não fosse finalizada sem reforço. Scheckel fixou a duração da tentativa e do atraso máximo que poderia ser alcançado. $\mathrm{O}$ step size e o IET foram variáveis. Em Cumming e Berryman a duração da tentativa dependia das latências das respostas no modelo e nos estímulos comparações, o step size e o IET foram fixados em $0,5 \mathrm{~s}$ e $25 \mathrm{~s}$, respectivamente. Tanto os pombos de Cumming e Berryman quanto os macacos de Scheckel realizaram as discriminações condicionais de identidade com atraso, e foram verificados valores limites diferentes para cada espécie e redução da variabilidade do atraso ajustado com o aumento da exposição ao procedimento. Apesar de não terem realizado nenhuma manipulação experimental, os dois estudos foram importantes para demonstrar o fenômeno em duas espécies e a quantificação do intervalo de retenção com manutenção da precisão do responder. 


\section{Tabela 1}

Variáveis, Parâmetros e Critérios Utilizados em Estudos Pioneiros que Utilizaram TDMTS elou Manipularam Variáveis Metodológicas

\begin{tabular}{|c|c|c|c|c|c|c|c|c|c|c|}
\hline $\begin{array}{l}\text { Identificação do } \\
\text { Estudo }\end{array}$ & VI & Sujeitos (n) & DMTS & Estímulos & $\begin{array}{c}\text { FR } \\
\text { Modelo }\end{array}$ & $\begin{array}{c}\text { Atraso } \\
\text { Inicial (s) }\end{array}$ & $\begin{array}{l}\text { Atraso } \\
\text { Máximo } \\
\text { (s) }\end{array}$ & IET (s) & $\begin{array}{l}\text { Critério de ajuste } \\
\text { do atraso }\end{array}$ & $\begin{array}{l}\text { Step size } \\
\quad(\mathrm{ms})\end{array}$ \\
\hline $\begin{array}{l}\text { Cumming e Berryman } \\
\text { (1965) }\end{array}$ & Sem manipulação & Pombo (1a) & Identidade & Visual (cor) & FR 5 & 0,5 & Não & 25 & A: $2 ; \mathrm{D}: 1$ & 500 \\
\hline Scheckel (1965) & Sem manipulação & Macaco (4) & Identidade & Visual (cor) & FR 1 & 1 & 105 & Variável & A: $2 ; \mathrm{D}: 1$ & Variável \\
\hline Jarrard e Moise (1970) & $\begin{array}{l}\text { Restrição física } \\
\text { (com x sem) }\end{array}$ & Macaco (3) & Identidade & Visual (cor) & FR 1 & 0 & Limit.b & 8 & A: $3 ; \mathrm{D}: 2$ & 2500 \\
\hline Sidman et al. (1971) & $\begin{array}{l}\text { Caracterização de } \\
\text { Repertório }\end{array}$ & Afásico (1) & $\begin{array}{l}\text { Identidade } \\
\text { e arbitrário }\end{array}$ & $\begin{array}{l}\text { Visual, } \\
\text { auditivo e } \\
\text { tatil }\end{array}$ & FR 1 & 0 & 40 & 1,5 & A: $1 ; \mathrm{D}: 1$ & 4000 \\
\hline Ferraro et al. (1971) & $\begin{array}{l}\text { Idade ( } 40 \text { a } 130 \\
\text { meses) }\end{array}$ & Criança (40) & Identidade & Visual (cor) & FR 1 & 2 & 60 & 3 & A: $2 ; \mathrm{D}: 1$ & 2000 \\
\hline $\begin{array}{l}\text { Wenger e Kimball } \\
\text { (1992) }\end{array}$ & $\begin{array}{l}\text {.IET } \\
\text {.Drogas de abuso }\end{array}$ & Pombo (5) & Identidade & Visual (cor) & FR 15 & 3 & Limit.b & 0 a 30 & $\begin{array}{l}\text { A: } 5 \\
\text { D: } 3 \text { ou mais }\end{array}$ & 3000 \\
\hline Wenger et al. (1993) & $\begin{array}{l}\text {.IET } \\
\text {.Diazepam, morfina, } \\
\text { escopolamina, } \\
\text { metilescopolamina, } \\
\text { fisostigmina e } \\
\text { neostigmina }\end{array}$ & Pombo (5) & Identidade & Visual (cor) & FR 15 & 3 & Limit.b & 10 e $30 \mathrm{c}$ & $\begin{array}{l}\text { A: } 5 \\
\text { D: } 2 \text { ou mais }\end{array}$ & 3000 \\
\hline Poling et al. (1996) & $\begin{array}{l}\text { Reforço (igual x } \\
\text { diferente) }\end{array}$ & Galinha (6) & Identidade & Visual (cor) & FR 5 & 0 & 60 & $\begin{array}{l}\text { S+:10 } \\
\text { S-: } 12,5\end{array}$ & A: $3 ; \mathrm{D}: 3$ & 1000 \\
\hline $\begin{array}{l}\text { Robinson et al. (2000), } \\
\text { Exp. } 2\end{array}$ & $\begin{array}{l}\text { Motivação (acesso à } \\
\text { água antes da } \\
\text { sessão) }\end{array}$ & Rato (14) & Oddity & $\begin{array}{l}\text { Visual } \\
\text { (Posição) }\end{array}$ & FR 1 & 1 & 29 & 10 & $\begin{array}{l}\text { A: maior que } \\
75 \% \\
\text { D: } 25 \% \text { ou mais }\end{array}$ & 2000 \\
\hline $\begin{array}{l}\text { Buccafusco et al. } \\
\text { (2002) }\end{array}$ & $\begin{array}{l}\text {.Idade } \\
\text {.Esopolamina }\end{array}$ & $\begin{array}{l}\text { Macaco } \\
\text { adulto (6) e } \\
\text { idoso (10) }\end{array}$ & Identidade & Visual (cor) & FR 1 & 0 & Limit.b & 5 & A: $1 ; \mathrm{D}: 1$ & 1000 \\
\hline
\end{tabular}


MTS COM AJUSTE DO ATRASO

\begin{tabular}{|c|c|c|c|c|c|c|c|c|c|c|}
\hline Porritt e Poling (2008) & $\begin{array}{l}\text { Step-size } \\
\text {.Escopolamina }\end{array}$ & Rato (7) & Oddity & $\begin{array}{l}\text { Visual } \\
\text { (Posição) }\end{array}$ & FR 1 & 0 & Limit.b & 5 & $\begin{array}{l}\text { A: } 3 \text { ou } 1 \\
\text { D: } 3 \text { ou } 1\end{array}$ & $\begin{array}{l}200,500 \\
1000 \mathrm{e} \\
2000\end{array}$ \\
\hline $\begin{array}{l}\text { Kangas et al. (2010), } \\
\text { Exp. } 1\end{array}$ & FR no modelo & Pombo (4) & Identidade & Visual (cor) & $\begin{array}{l}\text { FR } 1 \text { a } \\
32\end{array}$ & $0 \mathrm{~d}$ & Não & $\begin{array}{l}\text { S+: } 10 \\
\text { S-: } 13\end{array}$ & A: $2 ; \mathrm{D}: 1$ & 1000 \\
\hline $\begin{array}{l}\text { Kangas et al. (2010), } \\
\text { Exp. } 2\end{array}$ & $\begin{array}{l}\text { Atraso inicial } \\
\text { (sessão) }\end{array}$ & Pombo (4) & Identidade & Visual (cor) & FR 16 & $\begin{array}{l}0 \\
2 \mathrm{x} \mathrm{d}_{\mathrm{d}}\end{array}$ & Não & $\begin{array}{l}\text { S+: } 10 \\
\text { S-: } 13\end{array}$ & A: $2 ; \mathrm{D}: 1$ & 1000 \\
\hline $\begin{array}{l}\text { Kangas et al. (2010), } \\
\text { Exp. } 3\end{array}$ & Step size & Pombo (4) & Identidade & Visual (cor) & FR 16 & $0 \mathrm{~d}$ & Não & $\begin{array}{l}\text { S+: } 10 \\
\text { S-: } 13\end{array}$ & A: $2 ; \mathrm{D}: 1$ & $\begin{array}{l}1000 \\
2000\end{array}$ \\
\hline $\begin{array}{l}\text { Eilifsen e Arntzen } \\
\text { (2011), Exp. } 1\end{array}$ & $\begin{array}{l}\text { Atraso inicial } \\
\text { (condição) }\end{array}$ & Adulto (10) & Arbitrário & $\begin{array}{l}\text { Visual } \\
\text { (símbolos) }\end{array}$ & FR 1 & $\begin{array}{l}0 \\
5\end{array}$ & $\begin{array}{l}3 \\
8\end{array}$ & 0,5 & $\begin{array}{l}\text { A: } 90 \% \text { no bloco; } \\
\text { D: } 2\end{array}$ & 250 \\
\hline Friedel (2011), Exp 1 & FR no modelo & Pombo (4) & Identidade & Visual (cor) & $\begin{array}{l}\text { FR } 1 \text { a } \\
16\end{array}$ & $0 \mathrm{~d}$ & Não & 10 & A: $6 ; \mathrm{D}: 1$ & 1000 \\
\hline Friedel (2011), Exp 2 & Critério de ajuste & Pombo (4) & Identidade & Visual (cor) & $\begin{array}{l}\text { FR } 8 \text { ou } \\
16\end{array}$ & $0 \mathrm{~d}$ & Não & 10 & A: 6; D: 1 e 2 & 1000 \\
\hline Friedel (2011), Exp 3 & $\begin{array}{l}\text { Atraso inicial } \\
\text { (sessão) }\end{array}$ & Pombo (4) & Identidade & Visual (cor) & $\begin{array}{l}\text { FR } 8 \text { ou } \\
16\end{array}$ & $\begin{array}{l}0 \\
\mathrm{X} \mathrm{d}\end{array}$ & Não & 10 & A: $6 ; \mathrm{D}: 2$ & 1000 \\
\hline $\begin{array}{l}\text { Lian e Arntzen (2011), } \\
\text { Exp. } 1 \text { e } 2\end{array}$ & $\begin{array}{l}\text { MTS com atraso } \\
\text { fixo vs. MTS com } \\
\text { ajuste }\end{array}$ & Criança (20) & Arbitrário & $\begin{array}{l}\text { Visual } \\
\text { (forma } \\
\text { abstrata) }\end{array}$ & FR 1 & 0 & $\begin{array}{l}0,1 \\
3 \\
6\end{array}$ & 0,5 & $\begin{array}{l}\text { A: } 80 \% \text { no bloco; } \\
\text { D: mais de } 20 \%\end{array}$ & $\begin{array}{l}8 \\
250 \\
500\end{array}$ \\
\hline $\begin{array}{l}\text { Lian e Arntzen (2011), } \\
\text { Exp. } 3\end{array}$ & $\begin{array}{l}\text { Atraso inicial } \\
\text { (condição) }\end{array}$ & Criança (10) & Arbitrário & $\begin{array}{l}\text { Visual } \\
\text { (forma } \\
\text { abstrata) }\end{array}$ & FR 1 & $\begin{array}{l}0 \\
1,5\end{array}$ & 3 & 0,5 & $\begin{array}{l}\text { A: } 80 \% \text { no bloco; } \\
\text { D: mais de } 20 \%\end{array}$ & 125 \\
\hline $\begin{array}{l}\text { Arntzen e } \\
\text { Steingrimsdottir (2014) }\end{array}$ & Step size & $\begin{array}{l}\text { Idoso com } \\
\text { demência (1) }\end{array}$ & Identidade & $\begin{array}{l}\text { Visual } \\
\text { (forma } \\
\text { abstrata) }\end{array}$ & FR 1 & 0 & Não & 2 & $\begin{array}{l}\text { A: } 100 \% \text { no } \\
\text { bloco; D: } 1\end{array}$ & $\begin{array}{l}100 \text { ou } \\
500\end{array}$ \\
\hline
\end{tabular}

Nota. Critério de ajuste: A=número ou \% de acertos em um bloco de tentativas para aumentar o atraso; $\mathrm{D}=$ número ou \% de erros no bloco para diminuir o atraso a Autor menciona vários sujeitos, mas relata os resultados de apenas um

b Atraso limitado pelo máximo de tentativas e/ou pelo tempo de sessão

c IET de $10 \mathrm{~s}$ foi usado para diazepam e morfina; para as demais drogas foram utilizados os dois valores de IET

d Atraso inicial da sessão dependia do atraso final da sessão anterior 
Sidman et al. (1971) realizaram um estudo, com um idoso afásico, que descreve técnicas comportamentais para examinar, avaliar e classificar déficits de afasia, envolvendo vários procedimentos, respostas e estímulos. $\mathrm{O}$ TDMTS foi um dos procedimentos utilizados, mas semelhante à Cumming e Berryman (1965) e Scheckel (1965), não houve manipulação experimental. O objetivo foi demonstrar a importância de uma metodologia diversificada e a partir de análises funcionais para avaliar déficits comportamentais em populações clínicas. Desta maneira, o estudo também pode ser considerado uma demonstração pioneira com MTS arbitrário (quando as relações ensinadas são definidas pelo experimentador e não por semelhança física entre os estímulos, e.g., palavra ditada e palavra escrita), com estímulos auditivos e táteis, e utilizando parâmetros do procedimento diferentes daqueles utilizados por Cumming e Berryman (1965) e Scheckel (1965).

Considerando todos os experimentos da Tabela 1, observa-se variação entre eles em todos os aspectos metodológicos listados. As análises que serão feitas agora resumirão as diferenças em cada aspecto e descreverão resultados de pesquisas que manipularam o aspecto em foco.

\section{Sujeitos dos Estudos com TDMTS}

Observa-se na terceira coluna da Tabela 1 que o TDMTS foi utilizado com diferentes sujeitos: pombos, galinhas, ratos, macacos e humanos. Estudos com humanos foram realizados com crianças, adultos e idosos (Arntzen \& Steingrimsdottir, 2014; Eilifsen \& Arntzen, 2011; Ferraro, Francis, \& Perkins, 1971; Lian \& Arntzen, 2011). Ferraro et al. (1971) investigaram diferenças no atraso alcançado no TDMTS de identidade de cor relacionadas às faixas etárias das crianças. As crianças mais novas (51-60 meses) não foram capazes de responder corretamente quando o atraso foi superior a $0 \mathrm{~s}$. Crianças mais velhas (121-130 meses), por sua vez, responderam corretamente mesmo com atrasos superiores a 40 s. Um estudo com primatas não humanos (macaques) adultos e idosos (Buccafusco, Terry Jr., \& Murdoch, 2002) mostrou que os atrasos ajustados médios e máximos diminuíram drasticamente com a idade. Não foram identificados estudos com manipulação ampla de idade com humanos.

\section{Tipos de Discriminações Condicionais}

As colunas 4 e 5 da Tabela 1 apresentam os tipos de discriminações condicionais que foram ensinadas com o TDMTS e de estímulos, respectivamente. Relações de identidade de cor ou forma, ou seja, a escolha definida como correta é a do estímulo de comparação com cor ou forma igual a do modelo, foram requeridas em 13 dos 21 experimentos. Dois estudos (Porritt \& Poling, 2008; Robinson, Scheff, Pierre-Louis, \& Han, 2000) utilizaram tarefas de escolha da posição diferente (oddity ou non matching) e três o MTS arbitrário. Neste último, o modelo e o estímulo de comparação correspondente não apresentam semelhança física; a relação é definida pelo experimentador (e.g., uma forma abstrata e um som). A aprendizagem de relações de identidade é um pré-requisito para relações arbitrárias (Sidman, 1994), o que sugere que as últimas são relações mais complexas. No estudo de Sidman et al. (1971), a avaliação do repertório do participante afásico incluiu relações de identidade e arbitrárias. A inclusão dos dois tipos de relações em uma situação experimental precisa, entretanto, ser cautelosa uma vez que participantes podem continuar respondendo sob controle de similaridades físicas entre os estímulos na tarefa de MTS arbitrário após realizar a tarefa de MTS de identidade (Sidman, 1994). Este efeito de ordem já foi relatado (Steingrimsdottir \& Arntzen, 2011) em paciente diagnosticado com demência, mas não para adultos saudáveis (Steingrimsdottir \& Arntzen, 2014).

\section{Razão Fixa no Modelo}

$\mathrm{O}$ número de respostas (FR) exigido no modelo (também chamado de respostas para retirar o modelo; Wenger \& Kimball, 1992) variou de 1 a 32 entre os estudos (Coluna 6 da Tabela 1), com predominância do uso de FR 1. Os dois estudos que manipularam esta variável (Friedel, 2011; Kangas et al., 2010) concluíram que os valores de atrasos ajustados médios aumentam em função do aumento da exigência de resposta aos estímulos modelo, seguindo uma função curvilínea positivamente acelerada. Uma vez que a resposta no modelo se mostrou ser um fator importante para definir o valor final do atraso ajustado, seria importante obter resultados adicionais com outras espécies além de pombos.

\section{Atraso Inicial}

No processo de aquisição de discriminações, a proximidade temporal do modelo e dos estímulos de comparação é crucial para a aprendizagem (Cumming \& Berryman, 1965). Nos experimentos analisados, o atraso definido no início da condição de TDMTS variou de 0 a $5 \mathrm{~s}$, com predominância do uso de $0 \mathrm{~s}$. Apenas um dos estudos variou o atraso inicial da condição (Lian \& Arntzen, 2011, dados do Experimento 3 comparados com Experimento 2) e mostrou que mudar de $0 \mathrm{~s}$ para 1,5 s o atraso inicial facilitou o ensino das relações arbitrárias (menor número de tentativas) para crianças de seis a nove anos. Entretanto, neste estudo a medida comportamental não foi o atraso ajustado, uma vez que o atraso final era curto e pré-definido. Considerando que a mudança de MTS simultâneo para DMTS com atraso zero (modelo não está mais presente no momento da escolha) já pode representar uma quebra no desempenho (Cumming \& Berryman, 1965), especialmente em crianças e animais não humanos, seria importante a realização de estudos para investigar o efeito desta variável no ajuste do atraso.

Quando mais de uma sessão é realizada com TDMTS, é necessário definir o atraso a cada sessão. Nos experimentos analisados, dois critérios foram utilizados: restabelecer o atraso inicial em cada sessão ou utilizar algum valor próximo ou igual ao do final da sessão anterior (Coluna 6, indicado pelo sobrescrito "a"). Kangas et al. (2010, Experimento 2) e Friedel (2011, Experimento 3) investigaram o efeito desta variável com pombos e relataram atrasos ajustados mais longos quando a sessão é iniciada com base no valor dos atrasos finais da sessão anterior. Realizar o ajuste restabelecendo o atraso inicial em cada sessão, pode ser uma forma de controlar parâmetros da sessão, importante para determinados objetivos de pesquisa (Kangas et al.). Este critério, entretanto, limita a variação dos atrasos, considerando que as sessões terminam com um número fixo de tentativas ou 
por tempo. Mesmo que sejam utilizadas sessões longas, os atrasos mais longos serão testados somente quando provavelmente estejam ocorrendo efeitos de fadiga e saciação.

\section{Atraso Máximo}

No primeiro contato com o TDMTS é natural pensar que, sendo um procedimento de ajuste do atraso, o resultado seria identificar o maior valor de atraso que gerasse desempenho preciso, considerando algum critério. O atraso máximo neste caso dependeria, então, somente do desempenho do sujeito. A análise dos experimentos listados na Tabela 1 (Coluna 7) sugere algo diferente. Oito experimentos fixaram o atraso final. Isto significa que o valor do atraso não aumentava se o participante atingisse o valor definido. Um experimento utilizou este critério para comparar o desempenho em TDMTS com MTS com atraso fixo (Lian \& Arntzen, 2011, Experimento 2); outros (Eilifsen \& Arntzen, 2011; Lian \& Arntzen, 2011, Experimento 3) por utilizarem como variável dependente o número de tentativas e não o atraso ajustado. A definição do atraso máximo nos demais experimentos que fixaram o valor (Ferraro et al., 1971; Poling et al., 1996; Robinson et al., 2000 - Experimento 2; Scheckel, 1965; Sidman et al., 1971) pode não ter sido arbitrária, mas baseada em estudos anteriores ou em outras características do procedimento (e.g., duração da sessão, número de tentativas).

Cinco estudos limitaram indiretamente o valor do atraso máximo (indicados por "Limit" na Tabela 1) ao utilizar o critério de restaurar o valor inicial no início de cada sessão e fixar o tempo ou número de tentativas da sessão. Os demais oito estudos não limitaram, nem fixaram os valores do atraso ajustado. Em estudos que utilizam o atraso ajustado como VD, é importante não limitar ou fixar o seu valor máximo.

A definição do atraso máximo levanta também a questão do critério de estabilidade ou do cálculo utilizado para definir o atraso ajustado final baseado no desempenho. Este é outro aspecto que varia entre os estudos, que utilizaram tanto a média de um bloco de tentativas que atinge um critério de estabilidade, a média de todos os valores, ou o valor máximo, para citar alguns. A variação desses critérios leva, muito provavelmente a atrasos finais diferentes (Hanna \& Blackman, 2005).

\section{Intervalo entre Tentativas (IET)}

Intervalo entre tentativas são geralmente utilizados em procedimentos de tentativas discretas para reduzir o efeito da tentativa anterior sobre o desempenho na tentativa corrente em DMTS (e.g., Bigelow \& Poremba, 2013). Todos os estudos analisados utilizaram IET. Os intervalos variaram entre 0 e $30 \mathrm{~s}$, sendo os intervalos até $10 \mathrm{~s}$ mais frequentes (12 experimentos). Os dois estudos desta revisão que manipularam o IET (Wenger, Hudzik, \& Wright, 1993; Wenger \& Kimball, 1992) não relataram efeito da variação do IET. Dois estudos (Kangas et al., 2010; Poling et al., 1996) utilizaram IETs diferentes para tentativas com respostas corretas e incorretas. Este pode ser um controle importante para equiparar a duração programada das tentativas, descontando-se o período de acesso ao reforço.

\section{Critério de Ajuste do Atraso}

O ajuste do atraso no TDMTS é realizado com base em número ou porcentagem de acertos (aumento) e de erros (diminuição) em blocos ou conjuntos de tentativas. $\mathrm{O}$ critério para definir o ajuste do atraso é outro parâmetro que variou bastante entre os estudos analisados (Coluna 10, Tabela 1). Para aumentar o atraso, foram utilizados de uma a seis respostas corretas ou porcentagens de acerto maiores que 75 . Para diminuir o atraso, um a três erros ou porcentagem de erros igual ou maior que 25. Nota-se que quanto maior o número de erros permitido (e menor a quantidade mínima de acertos), menos diminuição do atraso pode ocorrer, podendo refletir no atraso ajustado final. Friedel (2011), utilizando pombos, manipulou o critério de ajuste em duas condições. Na condição mais restritiva, o atraso era aumentado se seis acertos consecutivos ocorressem e diminuído se um erro ocorresse. $\mathrm{Na}$ condição mais tolerante, a mesma exigência para aumento foi utilizada, mas o atraso diminuía apenas com a ocorrência de dois erros. Observaram-se atrasos ajustados maiores na condição mais tolerante (dois erros).

Destaca-se que o critério de ajuste do atraso pode ter relação com outras variáveis do procedimento, como o tamanho do bloco de tentativas ou o número de discriminações condicionais requeridas na tarefa. Para se configurar um procedimento de ensino de discriminação condicional, pelo menos duas relações/discriminações devem ser ensinadas (e.g., vermelho-vermelho e amarelo-amarelo). A maioria dos estudos com animais não humanos utilizou duas discriminações de cores e posições, porém os estudos com humanos (Arntzen \& Steingrimsdottir, 2014; Elifisen \& Arntzen, 2011; Lian \& Arntzen, 2011; Sidman et al., 1971) utilizaram três ou mais. Como o critério é baseado na acurácia das respostas, a quantidade e complexidade das discriminações devem ter influenciado os resultados.

\section{Step Size}

Além do critério para definir quando ajustar o atraso, o pesquisador define quanto de alteração deve ser feita. $\mathrm{O}$ tamanho do incremento ou decremento do ajuste (step size) variou de 8 a $4000 \mathrm{~ms}$ entre os estudos (Coluna 11, Tabela 1). O step size foi manipulado em três experimentos (Arntzen \& Steingrimsdottir, 2014; Kangas et al., 2010, Experimento 3; Porrit \& Poling, 2008). Porrit e Poling (2008) utilizaram quatro valores de step size $(0,2,0,5,1$ e $2 \mathrm{~s})$, em um estudo com ratos que aprenderam a escolher a posição diferente do modelo. Os autores relataram que a acurácia e o número de tentativas realizadas até o critério diminuíram com o aumento do step size, e o maior valor de atraso alcançado aumentou com o aumento do step size. Kangas et al. (2010, Experimento 3) utilizaram step sizes de $1 \mathrm{~s}$ e $2 \mathrm{~s}$ em uma tarefa de escolha da cor igual ao modelo, com pombos. As médias de atraso ajustado não variaram sistematicamente entre condições. No entanto, a variabilidade aumentou consideravelmente na condição com step size maior. A amplitude média dos atrasos intrasessão (i.e., a diferença entre os valores máximo e mínimo por sessão) praticamente dobrou, quando o step size dobrou. $\mathrm{O}$ estudo de Arntzen e Steingrimsdottir (2014) investigou o efeito de step sizes 0,1 e $0,5 \mathrm{~s}$ no desempenho de discriminações condicionais de identidade de formas abstratas, com um homem de 62 anos, diagnosticado com demência. Foi 
identificado o valor máximo de atraso em que o participante fosse capaz de manter acurácia nas tentativas de discriminação com três diferentes estímulos modelos. O menor step size: (a) produziu menos variabilidade nos atrasos ajustados, (b) conduziu ao alcance de maiores valores do atraso, mas (c) ao menor valor do atraso final, quando o critério de estabilidade foi atingido.

Os estudos que manipularam o step size encontraram resultados semelhantes, com a menor variabilidade observada com step size menor (Arntzen \& Steingrimsdottir, 2014; Kangas et al., 2010) e maior atraso ajustado com step size maior (Arntzen \& Steingrimsdottir, 2014; Porrit \& Poling, 2008). A diferença do menor valor do atraso final (Arntzen \& Steingrimsdottir, 2014; Kangas et al., 2010) pode ser devida a outras variáveis de procedimento que os estudos não compartilharam, como a contingência de resposta no modelo mais exigente, FR 6 (Kangas et al., 2010) e FR 1 (Arntzen \& Steingrimsdottir, 2014).

Além da programação de step size com valor fixo, também foi utilizado step size variável (Scheckel, 1965), mas não foram encontrados estudos que comparam diferentes programações do step size.

\section{Outras Manipulações}

No contexto das pesquisas analisadas devem-se verificar empiricamente possíveis diferenças entre o desempenho em MTS com atraso fixo e variável. Lian e Arntzen (2011, Experimentos 1 e 2) compararam o FDMTS com TDMTS para estabelecer discriminação condicional e equivalência de estímulos (MTS arbitrário com figuras abstratas) com 20 crianças com idades entre seis e nove anos. Os atrasos foram de $0 \mathrm{~s}, 1 \mathrm{~s}, 3 \mathrm{~s}$ e $6 \mathrm{~s}$. Os resultados mostraram que a aprendizagem foi mais lenta e o desempenho inferior em testes com atraso maior e com TDMTS do que com FDMTS utilizados nos treinos de relações de linha de base.

Poling et al. (1996) investigaram se a programação de reforços sistematicamente diferentes para cada relação ensinada aumentava a acurácia. Em um procedimento de TDMTS de identidade de cor, seis galinhas domésticas foram expostas às condições diferencial e não diferencial. Na condição diferencial, respostas corretas em uma cor tinham como consequência $1 \mathrm{~s}$ de acesso ao alimento e respostas corretas na outra cor eram seguidas por $4 \mathrm{~s}$ de acesso ao alimento. Na condição não diferencial, 1 ou $4 \mathrm{~s}$ era aleatoriamente programado ao longo das tentativas. Os resultados mostraram maior acurácia e atrasos mais longos nas sessões finais da condição diferencial, quando comparado com as sessões finais da condição não diferencial.

Ao investigar tarefas de discriminação condicional com primatas especulava-se que o desempenho poderia ser afetado pelo engajamento desses animais em respostas hipoteticamente incompatíveis com a realização da tarefa (e.g., movendose no compartimento de testagem). Para investigar essa variável relacionada com a programação da contingência, Jarrard e Moise (1970) testaram três primatas (macaque) em um procedimento de TDMTS de identidade sob duas condições: com e sem restrição física. Segundo os autores, os resultados indicaram que a inibição de respostas incompatíveis não leva a uma melhora do desempenho na tarefa experimental, uma vez que não houve diferença significativa entre as condições de restrição e não-restrição.

\section{CONCLUSÃO E CONSIDERAÇÕES FINAIS}

O procedimento TDMTS permite identificar intervalos de retenção individuais (i.e., os valores de atraso que o desempenho mantém precisão para cada sujeito). A partir desses intervalos, a curva de esquecimento/recordação é obtida (Van Hest \& Steckler, 1996). Muitos aspectos podem, entretanto, variar entre os estudos, dificultando o trabalho do pesquisador, tanto para programar um experimento, quanto na comparação entre estudos. Esta revisão mostrou os critérios e valores de parâmetros mais utilizados na literatura, e resumiu os efeitos da manipulação da maioria dos aspectos metodológicos. As evidências principais encontradas até fevereiro de 2019 foram: (a) atrasos médios mais longos foram obtidos com o aumento da observação ao modelo (Friedel, 2011; Kangas et al., 2010); (b) a programação do atraso inicial de cada sessão baseada nos atrasos finais da sessão anterior levou a atrasos ajustados finais mais longos (Kangas et al., 2010; Friedel, 2011); (c) a variação no tamanho do IET não influenciou os atrasos ajustados (Wenger et al., 1993; Wenger \& Kimball, 1992); (d) critério de ajuste mais tolerante produziu atrasos finais mais longos (Friedel, 2011); (e) o tamanho do incremento definido para o ajuste do atraso (step size) é diretamente relacionado à variabilidade dos atrasos ajustados e ao valor máximo do atraso intrasessão (Arntzen \& Steingrimsdottir, 2014; Kangas et al., 2010; Porrit \& Poling, 2008); (f) a programação de reforços diferenciais para cada discriminação produziu atrasos ajustados mais longos (Poling et al., 1996). Estes resultados carecem de replicações controladas para avaliar a generalidade em um contexto com tamanha variação de parâmetros, critérios e sujeitos experimentais.

Muitos dos estudos revisados com o TDMTS são investigações sobre fenômenos relacionados à memória. A maioria dos estudos utilizou animais não humanos como sujeitos, apesar da importância central do conceito "para a vida humana, em habilidades como fala, compreensão de texto, prospecção e planejamento futuro" (Carruthers, 2013, p. 10371). Pode ser utilizado também para comparar memória de trabalho entre diferentes espécies e para quantificar diferenças em função do desenvolvimento, se utilizado com participantes de diferentes faixas etárias.

Todos os estudos utilizaram estímulos visuais e apenas Sidman et al. (1971) incluiu estímulos de outras modalidades. Uma vez que cada espécie tem uma modalidade sensorial dominante (Thompson, 1981), seria relevante comparar curvas de esquecimento com estímulos de modalidade diferentes. Além disso, existem evidências com outras metodologias de que determinados estímulos (e.g., música) favorecem o lembrar em pacientes com demência (e.g., Foster \& Valentine, 2001). Evidência 
adicional com TDMTS sobre esta questão pode ser um achado importante.

Estudos com TDMTS, embora minoritários na literatura, merecem atenção especial, pois, às vezes, relatam um desempenho impressionante de alcance do atraso (Lind et al., 2015). É um procedimento que pode ser indicado para o treinamento da memória, o que vem da prática dos participantes iniciarem em uma tarefa com atraso zero e em seguida são expostos a atrasos progressivamente mais longos à medida que o desempenho melhora (e.g., Arntzen $\&$ Steingrimsdottir, 2014). Além de possibilitar a criação de sessões personalizadas para diferentes espécies, tipos e graus de comprometimentos cognitivos.

\section{DECLARAÇÃO DE CONFLITO DE INTERESSES}

Os autores declaram que não há conflito de interesses relativos à publicação do presente artigo.

\section{CONTRIBUIÇÃO DE CADA AUTOR}

Certificamos que as duas autoras participaram suficientemente do trabalho para tornar pública sua responsabilidade pelo conteúdo. As autoras atuaram de forma compartilhada na concepção, revisão bibliográfica e redação do artigo.

\section{DIREITOS AUTORAIS}

Este é um artigo aberto e pode ser reproduzido livremente, distribuído, transmitido ou modificado, por qualquer pessoa desde que usado sem fins comerciais. $\mathrm{O}$ trabalho é disponibilizado sob a licença Creative Commons 4.0 BY-NC.

\section{(cc) BY-NC}

\section{REFERÊNCIAS}

Arntzen, E., \& Steingrimsdottir, H. S. (2014). On the use of variations in a delayed matching-to-sample procedure in a patient with neurocognitive disorder. In M. H. Swahn, J. B. Palmier, \& S. M. Braunstein (Eds.), Mental disorder (pp. 123-138). iConcept Press Ltd. Recuperado

https://www.iconceptpress.com/books/mentaldisorder/01-0042-001/

Bigelow, J., \& Poremba, A. (2013). Auditory proactive interference in monkeys: The role of stimulus set size and intertrial interval. Learning and Behavior, 41(13), 319-332. doi: 10.3758/s13420-013-0107-9

Blough, D. (1959). Delayed matching in the pigeon. Journal of the Experimental Analysis of Behavior, 2, 151-160. doi: 10.1901/jeab.1959.2-151

Buccafusco, J. J., Terry Jr., A. V., Murdoch, P. B. (2002). A computer-assisted cognitive test battery for aged monkeys. Journal of Molecular Neuroscience, 19, 179185. doi: 10.1007/s12031-002-0030-6

Carruthers, P. (2013). Evolution of working memory. Proceedings of the National Academy of Sciences, 110(2), 10371-10378. doi: 10.1073/pnas.1301195110

Catania, A. C. (1999). Aprendizagem: Linguagem, comportamento e cognição. Porto Alegre: Artmed.

Cumming, W. W., \& Berryman, R. (1965). The complex discriminated operant: Studies of matching-to-sample. In D. I. Mostofsky (Org.), Stimulus generalization (pp. 284-330). Stanford, CA: Stanford University Press.

Dube, W. V., Balsamo, L. M., Fowler, T. R., Dickson, C. A., Lombard, K. M., \& Tomanari, G. Y. (2006). Observing behavior topography in delayed matching to multiple samples. The Psychological Record, 56, 233244. doi: 10.1016/j.jadohealth.2005.06.006

Eilifsen, C., \& Arntzen, E. (2011). Single-subject withdrawal designs in delayed matching-to-sample procedures. European Journal of Behavior Analysis, 12(1), 157-172. doi: 10.1080/15021149.2011.11434361

Ferraro, D. P., Francis, E. W., \& Perkins, J. J. (1971). Titrating delayed matching to sample in children. Developmental Psychology, 5(3), 488-493. doi: 10.1037/h0031598

Foster, N.A., Valentine, E.R. (2001). The effect of auditory stimulation on autobiographical recall in dementia. Experimental Aging Research, 27(3), 215-228. doi: 10.1080/036107301300208664

Friedel, J. E. (2011). An exploration of the titrating-delay match-to-sample procedure with pigeons (Dissertação de mestrado não publicada). University of North Texas, Denton, Texas. Recuperado de https:// digital.library.unt.edu/ark:/67531/metadc103316/m2/1/ high_res_d/thesis.pdf

Hanna, E. S., \& Blackman, D. E. (2005). Choice between differing delays and durations of reinforcement: on the generalization of preferences in discrete-trials to a freeoperant procedure. Brazilian Journal of Behavior Analysis, 1, 81-95. doi: 10.18542/rebac.v1i1.764

Jarrard, L. E., \& Moise, S. L. (1970). Short-term memory in the stumptail macaque: Effect of physical restraint of behavior on performance. Learning and Motivation, 1, 267-275. doi: 10.1016/0023-9690(70)90151-7

Kangas, B. D., Vaidya, M., \& Branch, M. N. (2010). Titrating-delay matching-to-sample in the pigeon. Journal of the Experimental Analysis of Behavior, 94, 69-81. doi: 10.1901/jeab.2010.94-69

Lian, T., \& Arntzen, E. (2011). Training conditional discriminations with fixed and titrated delayed matching-to-sample in children. European Journal of Behavior Analysis, 12(1), 173-193. doi: 10.1080/15021149.2011.11434362

Lind, J., Enquist, M., \& Ghirlanda, S. (2015). Animal memory: A review of delayed matching-to-sample data. Behavioural Processes, 117, 52-58. doi: 10.1016/j.beproc.2014.11.019

Mackay, H. A. (1991). Conditional stimulus control. In I. H. Iversen \& K. A. Lattal (Eds.), Techniques in the behavioral and neural sciences: Vol. 6. Experimental analysis of behavior (Part 1, pp. 301-350). Amsterdam: Elsevier.

Matos, M. A. (1981). O controle de estímulos sobre o comportamento. Psicologia, 7, 1-15.

Palmer, D. C. (1991). A behavioral interpretation of memory. In L. J. Hayes \& P. N. Chase (Eds.), Dialogues on verbal behavior: The first international institute on verbal relations (pp. 261-279). Reno, NV: Context Press. 
Poling, A., Temple, W., \& Foster, M. (1996). The differential outcomes effect: A demonstration in domestic chickens responding under a titrating-delayed-matching-to-sample procedure. Behavioural Processes, 36, 109-115. doi: 10.1016/0376-6357(95)00022-4

Porritt, M., \& Poling, A. (2008). Scopolamine effects under a titrating-delayed-nonmatching-to-position procedure. The Psychological Record, 58, 37-49. doi: 10.1007/BF03395601

Robinson, J. K., Scheff, A., Pierre-Louis, J., \& Han, C.J. (2000). Specificity of memory measures in an adjusting delayed nonmatching-to-position task for rats. Behavioural Brain Research, 111, 107-113. doi: 10.1016/S0166-4328(00)00149-2

Scheckel, C. L. (1965). Self-adjustment of the interval in delayed matching: Limit of delay in the rhesus monkey. Journal of Comparative and Physiological Psychology, 59, 415-418. doi: 10.1037/h0022058

Sidman, M. (1986). Functional analysis of emergent verbal classes. In T. Thompson \& M. D. Zeiler (Orgs.), Analysis and integration of behavioral units (pp. 213245). Hillsdale, NJ: Lawrence Erlbaum Associates.

Sidman, M. (1994). Equivalence relations and behavior: A research story. Boston: Authors Cooperative.

Sidman, M., Stoddard, L. T., Mohr, J. P., \& Leicester, J. (1971). Behavioral studies of aphasia: Methods of investigation and analysis. Neuropsychology, 9, 119140.

Steingrimsdottir, H. S. (2015). Learning and Remembering in Older Adults and Older Adults with Neurocognitive Disorders. (Tese de doutorado). Oslo and Akershus University College of Applied Sciences, Oslo, Noruega. Recuperado de https:// https://odahioa.archive.knowledgearc.net/handle/10642/2915

Steingrimsdottir, H. S., \& Arntzen, E. (2011). Using conditional discrimination procedures to study remembering in an Alzheimer's patient. Behavioral Interventions, 26,179-192. doi:10.1002/bin. 334

Steingrimsdottir, H. S., \& Arntzen, E. (2014). Performance by older adults on identity and arbitrary matching-tosample tasks. Psychological Record, 64, 827-839. doi: 10.1007/s40732-014-0053-8

Thompson, R. K. R. (1981). Nonconceptual auditory matching by a rhesus monkey reflects biological constraints on cognitive processes? Artigo apresentado na Northeastern Meeting of the Animal Behavior Society, Kingston, Ontario, Canada.

Van Hest, A., \& Steckler, T. (1996). Effects of procedural parameters on response accuracy: lessons from delayed (non-)matching procedures in animals. Cognitive Brain Research, 3(3-4), 193-203. doi: 10.1016/09266410(96)00006-7

Wenger, G. R., Hudzik, T. J., \& Wright, D. W. (1993). Titrating matching-to-sample performance in pigeons: Effects of diazepam, morphine, and cholinergic agents. Pharmacology Biochemistry and Behavior, 46(2), 435443. doi: 10.1016/0091-3057(93)90376-5

Wenger, G. R., \& Kimball, K. A. (1992). Titrating matching-to-sample performance: Effects of drugs of abuse and intertrial interval. Pharmacology Biochemistry and Behavior, 41(2), 283-288. doi: 10.1016/0091-3057(92)90099-2

Submetido em: 31/05/2019 Aceito em: 10/11/2019 